\title{
Integrating the transcriptome and proteome to identify important functional genes for laying hens with hard- or weak- shelled eggs
}

\section{Yan Wu}

Hubei Academy of Agricultural Sciences

Jinsong Pi ( $\sim$ pijinsong0523@163.com )

Hubei Academy of Agricultural Sciences

\section{Hao Zhang}

Hubei Academy of Agricultural Sciences

Hongwei Xiao

Hubei Academy of Agricultural Sciences

Ailuan Pan

Hubei Academy of Agricultural Sciences

Jie Shen

Hubei Academy of Agricultural Sciences

Yuejin Pu

Hubei Academy of Agricultural Sciences

\section{Zhenhua Liang}

Hubei Academy of Agricultural Sciences

Jinping Du

Hubei Academy of Agricultural Sciences

Research article

Keywords: Laying hen, Eggshell, Transcriptome, Proteome, lon transport

Posted Date: December 10th, 2020

DOI: https://doi.org/10.21203/rs.3.rs-124584/v1

License: (0) (i) This work is licensed under a Creative Commons Attribution 4.0 International License. Read Full License 


\section{Abstract \\ Background}

Eggshell strength and thickness are critical factors for reducing the egg breaking rate and preventing economic losses. The calcite biomineralization process is very important for eggshell quality. Therefore, we employed transcriptional sequencing and proteomics to investigate the differences between the uteruses of laying hens with high- or low-breaking-strength shells.

\section{Results}

We identified 1028 differentially expressed genes (DEGs) and 270 differentially expressed proteins (DEPs). The analysis results of GO terms and KEGG pathways showed most of the DEGs and DEPs were enriched in vital pathways related to such processes as calcium metabolism, hormone and amino acid biosynthesis, and cell proliferation and apoptosis. Some DEGs and DEPs that were coexpressed at mRNA and protein levels were verified.

\section{Conclusion}

in summary, this study confirmed that during normal calcification, there are differences in ion transport between the uterus of hens producing high-breaking-strength and those producing low-breaking-strength eggshells, which may help to elucidate the eggshell calcification process.

\section{Background}

The ability of chickens to produce high numbers of eggs over an extended laying cycle is very important for the poultry industry. The eggshells of birds play an important role in determining the physical and antimicrobial defenses of eggs and regulating the exchange of metabolic gases and water [1]. Eggshell quality is very important for hatchability [2], food safety and egg marketing [3]. Therefore, during the entire laying period, improving the sustainability of egg quality (especially eggshell strength) is very important for the poultry industry. Many factors, including health status, age, nutrition, and housing system, affect the productive performance and egg quality of laying hens [4]. Eggs with low eggshell strength are easily damaged during collection, storage, and transportation. On average, broken eggs account for $13-20 \%$ of eggs produced, which can lead to significant economic losses [5]. The eggshell is a complex structure with important biological functions for protection of the inner content of eggs after deposition, as well as for chicken embryo development [6]. The eggshell contains $95 \%$ calcium carbonate as polymorphic calcite and $3.5 \%$ organic matrix proteins [7]. The eggshell is formed in the uterus, which is a segment of the oviduct of laying hens. The process of eggshell formation can be divided into three crucial periods: initiation of crystal growth, linear crystal growth, and termination of mineralization [8]. The eggshell consists of a bilayered membrane, mammillary layer, palisade layer, vertical crystal layer and cuticle layer [9].

Previous studies have shown that high-strength eggshells and low-strength eggshells exhibits differences in the ultrastructure [10-12]. The ultrastructure of eggshells is related to matrix proteins that regulate morphology, growth kinetics and crystallographic orientation of calcite crystals $[13,14]$; these proteins include ovocleidin-116, ovocleidin-17, ovocalyxin-32, and ovocalyxin- 36 [15-18]. The matrix proteins have been shown to be involved in the biomineralization of the eggshell [19]. However, most of these discoveries provide only the genes expressed in certain segments of the chicken oviduct, the principal organ for egg development; consequently, the overall mechanisms of eggshell development have not been determined. Similar to other economically important traits, eggshell strengthening remains relevant throughout productive life of the laying hen and commonly deteriorates with age $[20,21]$. The decline in eggshell quality remains one of the major reasons for replacing commercial flocks [22]. Thus, elucidating the genetic basis and molecular mechanisms underlying eggshell strengthening at different chicken and laying stages is very important for breeders. Therefore, this study investigated the differences in the gene expression profiles of the uteri of laying hens producing high- or low-breaking-strength eggshells during peak laying periods. 


\section{Results}

\section{Analysis of the DEGs in the uterus for HE and LE hens}

A total of 353 million raw reads were obtained from the RNA-seq data. After the adapter and low-quality sequences were removed, clean data were obtained. The total number of reads generated from the six samples is shown in Supplementary Table S1. Each sample yielded more than 45 million raw reads. A total of 19128 expressed genes were detected between HE and LE hens. DEGs were identified by fold change $>1.5$ and P-values $<0.05$ (Supplementary Table S2). There were 1028 annotated genes that were significantly different in the uterus (Supplementary Table S3). Of these genes, 437 genes were upregulated, and 591 genes were downregulated (LE vs HE) (Fig. 1a).

GO analysis of the DEGs of the six uterus samples indicated that they were enriched in cellular component, biological process and molecular function for such terms as cell periphery, extracellular region, and actin cytoskeleton (Fig. 1b). The top 20 results from KEGG analysis showed that some vital pathways related to calcium metabolism, cell proliferation and apoptosis could also be influenced by these DEGs in the uterus, including the calcium signaling pathway, MAPK signaling pathway, and PPAR signaling pathway (Fig. 1c).

\section{Protein identifications and DEP analyses by TMT proteome}

To elucidate the mechanism underlying differences in eggshell strength, the differentially expressed proteins of the HE and LE groups were detected by TMT proteomics. In this experiment, 6786 proteins were identified. There were 270 differentially expressed proteins between the LE and HE groups that were significantly different (Supplement Table S4), including 161 upregulated proteins and 109 downregulated proteins (LE vs HE).

Analysis of these DEPs by GO analysis showed that the DEPs were primarily enriched in biological processes, molecular functions and cellular components including calcium ion binding, molecular function regulators, and extracellular regions (Fig. 2a). The top 20 results of KEGG enrichment analysis further indicated that these DEPs were most enriched in some vital pathways related to such processes as calcium metabolism, hormone and amino acid biosynthesis, and cell proliferation and apoptosis; these proteins were involved in the calcium signaling pathway, cell adhesion molecules (CAMs), ECM-receptor interactions, the p53 signaling pathway, biosynthesis of amino acids, and steroid hormone biosynthesis (Fig. 2b).

To further demonstrate the function of DEPs, we constructed a protein-protein interaction network of the DEPs (Fig. 2c). This study provides a new method and perspective for future research studying protein-protein interactions.

\section{Correlation between differentially expressed transcriptomes and proteomes}

To elucidate the relationship between the transcriptome and proteome in the HE and LE groups, two different omics datasets were integrated and analyzed. The results indicated that the number of DEGs and DEPs varied considerably in different eggshellstrength groups (Fig. 3a, 3b, 3c), and some genes (or proteins) were differentially expressed at the gene level but not at the protein level, possibly because they were late-response genes. There were 20 genes and proteins detected at both the gene and protein levels (Table 1). The GO enrichment analysis results showed that the correlated-expression DEGs/DEPs were primarily enriched in such processes as transporter activity, molecular function regulation, single-organism processes, membrane, and structural molecule activity (Fig. 3d); meanwhile, KEGG enrichment analysis results showed that the correlated-expression DEGs/DEPs were primarily enriched in such processes as metabolic pathways, the p53 signaling pathway, focal adhesion, the calcium signaling pathway, and SNARE interactions in vesicular transport(Fig. 3e). 
Table 1

The proteins/genes differentially expressed in both the proteome and transcriptome

\begin{tabular}{|c|c|c|c|c|}
\hline Gene_id & Tran_id & Prot_id & GO_fun & kegg \\
\hline $\mathrm{CDH} 17$ & 420225 & A0A1D5NVS2 & cell adhesion & - \\
\hline KRT14 & 408039 & A0A1D5PZ89 & $\begin{array}{l}\text { structural molecule } \\
\text { activity }\end{array}$ & - \\
\hline ANXA2 & 396297 & P17785 & $\begin{array}{l}\text { molecular function } \\
\text { regulator }\end{array}$ & - \\
\hline DKK3 & 396023 & F1NRD7 & extracellular region & - \\
\hline BET1 & 420563 & E1BRC4 & binding & SNARE interactions in vesicular transport(map04130) \\
\hline HTATIP2 & 424363 & A0A1L1RLC7 & - & - \\
\hline LOX & 396474 & Q05063 & $\begin{array}{l}\text { single-organism } \\
\text { process }\end{array}$ & - \\
\hline STAT1 & 424044 & Q5ZJK3 & $\begin{array}{l}\text { single-organism } \\
\text { process }\end{array}$ & $\begin{array}{l}\text { AGE-RAGE signaling pathway in diabetic } \\
\text { complications(map04933) }\end{array}$ \\
\hline PDLIM7 & 416362 & A0A1D5PJC9 & binding & - \\
\hline BPGM & 418172 & Q5ZHV4 & $\begin{array}{l}\text { single-organism } \\
\text { process }\end{array}$ & Metabolic pathways(map01100) \\
\hline TFF2 & 418534 & E1BZ37 & - & - \\
\hline CD109 & 421862 & F1NX21 & extracellular region & - \\
\hline $\mathrm{P} 2 \mathrm{RX} 7$ & 771952 & E1C6P3 & transporter activity & Calcium signaling pathway(map04020) \\
\hline RRM2B & 420253 & A0A1D5PI47 & $\begin{array}{l}\text { single-organism } \\
\text { process }\end{array}$ & p53 signaling pathway(map04115) \\
\hline FBLN1 & 373979 & F1NX60 & extracellular region & - \\
\hline ABCC3 & 422099 & F1NM51 & membrane & ABC transporters(map02010) \\
\hline SCIN & 420588 & A0A1I7Q413 & $\begin{array}{l}\text { single-organism } \\
\text { process }\end{array}$ & - \\
\hline COMP & 420120 & E1C8N1 & extracellular region & Focal adhesion(map04510) \\
\hline NT5DC3 & 427914 & A0A1D5PNR2 & - & - \\
\hline MAP2 & 424001 & A0A3Q2U4G6 & $\begin{array}{l}\text { single-organism } \\
\text { process }\end{array}$ & - \\
\hline
\end{tabular}

\section{The validation of differentially expressed genes and proteins}

In this study, the differentially expressed genes (DEGs) and differentially expressed proteins (DEPs) between LE and HE hens were identified. Seven upregulated and seven downregulated genes were randomly selected for verification via qPCR; eight proteins that were coexpressed at the mRNA and protein levels were randomly selected for verification by Western blotting. The results are shown in Fig. 4a and Fig. 4b. The log2 fold changes (LE/HE) were calculated based on the RNA-seq and qPCR results for DEGs, and the fold changes (LE/HE) were calculated based on the TMT and Western blotting results for DEPs. The expression trends indicated that the two methods produced consistent results. Additionally, we investigated the relative expression levels of KRT14, ANXA2 and DKK3 in different chicken tissues (i.e., heart, liver, spleen, lung, kidney, muscle and uterus) (Fig. 4c, 4d, 4e). In particular, the KRT14, ANXA2 and DKK3 genes exhibited high expression levels in the uterus compared with other tissues, and these genes were expressed at the lowest levels in the spleens and livers of hens. Thus, the tissue expression profile of the KRT14, ANXA2 and DKK3 genes demonstrated tissue specificity. 


\section{Discussion}

It is well-known that the formation of eggshells consists of several stages [23]. The formation of the mammillary knob layer follows the formation of the outer surface of the outer shell membrane and is the first calcified layer to be deposited, and its tips are embedded in the outer shell membrane [24]. The palisade layer formed with the mammillary knobs fuse, and the vertical crystalline layer is the last calcified layer to be deposited, consisting of short crystals running perpendicular to the shell membrane $[25,26]$. Previous studies have shown that shell quality is dependent on the mammillary layer [27], and another study suggested that the organization of the columns of the palisade layer is one of the major determinants of the rigidity of the shell and the strength and shell resistance of the eggs [28]. When eggshells are calcified in the uterus, large amounts of calcium ions and matrix proteins are required. Calcium metabolism and uterine proteins exhibit changes during the calcification periods (initiation, growth, and termination). Eggshell quality is determined by its ultrastructure [29], especially the palisade layer [30]. Therefore, many studies have focused on the growth period when the palisade layer is formed [31, 32].

Previous studies have shown that calcium could disrupt the endocrine system of females due to its wide spectrum of toxic effects on the uterus and mammary gland of rats and the developing human reproductive tract [33, 34]. Zhang et al. (2019)[35] reported that the initiation period of calcification determines eggshell strength. However, differences in the uterine transcriptome between hens laying eggs with high eggshell breaking strength and those producing eggs with low eggshell breaking strength during synchronous calcification periods have not been reported. In this study, the differences in gene expression and protein expression were identified in the uteri of LE and HE hens. The results showed that the numbers of DEPs were significantly lower than those of DEGs. Fewer proteins were detected than genes in the Venn diagram analysis, probably due to the short length of the sample treatment period, modification and activation at the protein level, or the limitations of protein detection technologies [36]. Previous studies have shown that most genes are divided into two categories according to their different activation pathways in response to stimuli. The early response genes were induced without de novo protein synthesis [37], and the late response genes were induced more slowly with de novo protein synthesis because this procedure requires signaling molecules or cytokines [38].

The DEGs and DEPs were classified according to KEGG analysis, and most DEGs and DEPs were determined to be enriched in vital pathways related to calcium metabolism, hormone or amino acid biosynthesis or cell proliferation or apoptosis. Thus, we hypothesized that the quality of eggshells is closely related to the deposition of calcium ions. GO analysis revealed that many DEGs and DEPs were enriched in ion transport functions or cytoskeletal functions associated with eggshell calcification. These results suggested that during normal calcification, there were differences in ion transport between the uteri of hens producing high- or low-breaking-strength eggshells, which helped to elucidate the eggshell calcification process. Aside from the common ion transport genes identified in previous reports, some novel genes were also described in this study (e.g., ANAX2, DKK3, and KRT14).

Annexin A2 (ANXA2) is a multifunctional calcium (Ca) and phospholipid-binding protein that is expressed in a wide spectrum of cells, including those participating in the inflammatory response [39]. Dickkopf 3 (Dkk3) is a secreted protein that belongs to the Dkk family and is encoded by the orthologous gene REIC. It was reported that $D k k 3$ is a physiological ER stressor in the mouse adrenal gland [40]. Keratin-14 (KRT14) is a key regulator of spheroid integrity, mesothelial attachment, and invasion into the submesothelial matrix. Keratin-14 (KRT14)-positive leader cells mediate mesothelial clearance and invasion by ovarian cancer cells [41]. In this study, ANXA2, KRT14 and DKK3 were determined to be significantly upregulated in LE hens compared to HE hens. In addition, the mRNA expression levels of the $A N X A 2, K R T 14$ and $D K K 3$ genes were observed significantly elevated in the uterus. In summary, our results indicate that $A N X A 2, K R T 14$ and $D K K 3$ may be related to the deposition of calcium in eggshells.

\section{Conclusions}

Based on the sequencing results and transcriptome and proteome analyses, our results were in keeping with previous findings indicating that the initiation period of calcification determines eggshell strength in the uterus. During normal calcification, differences in ion transport were observed between the uteri of hens producing high-breaking-strength eggshells and those producing low-breaking-strength eggshells, which may help to elucidate the eggshell calcification process.

\section{Methods}

Page 5/16 


\section{Ethics approval and consent to participate}

All animal experiments were conducted according to the guidelines established by the Regulations for the Administration of Affairs Concerning Experimental Animals (Ministry of Science and Technology, China, 2004). The tissues were collected from 43week-old hens. The hens were raised under free food intake and were humanely sacrificed in the laboratory.

\section{Samples collected}

Three eggs from 1820 hens (Jianghan chicken, which is 43-week-old and is a local chicken in China) were collected. Eggshell breaking strength, eggshell thickness and egg weight were assessed. The eggshell thicknesses of eggs were tested using an electronic digital micrometer (Deli, Deli Group Co., Ltd, China). Eggshell breaking strength was assessed using an eggshell strength meter (NFN388, FHK Fujipin Co., Ltd, Japan). Eggs were weighed using an electronic balance (ES-E, Tianjin de ante Sensing Technology Co., Ltd, Tianjin, China).

The uteri of hens producing eggs with extreme eggshell thickness and eggshell breaking strength were collected immediately after oviposition from six hens after surgery. There were three uterus samples from the hard-eggshell group (HE group, exhibiting strong and thick eggshells) and three uterus samples from the week-eggshell group (LE group, exhibiting weak and thin eggshell). Then, the uteri were frozen in liquid nitrogen and used for RNA-seq analysis and experimental validation.

\section{Transcriptome Sequencing}

Total RNA was isolated with TRIzol reagent (Invitrogen, USA). After total RNA was isolated from each individual sample and assessed, the concentration, quality and integrity were determined by a NanoDrop Spectrophotometer (NanoDrop Technologies, Wilmington, DE, USA). A total of $5 \mu \mathrm{g}$ RNA from each sample was employed for sequencing and library construction, which were performed at Novogene Biotech Co., Ltd. (Beijing, China).

The cDNA libraries were constructed using the TruSeq Stranded mRNA Library Prep Kit (Illumina, San Diego, CA, USA) according to the instructions of the manufacturer. Poly(A) mRNA was isolated from purified total RNA using biotin-oligo (dT) magnetic beads and fragmented to generate average insert sizes of approximately $350 \mathrm{bp}$ before the cDNA libraries were created. Quality control steps were undertaken using Qubit2.0 fluorescence spectrophotometry and an Agilent 2100 Bioanalyzer (Agilent Technologies, Palo Alto, CA, USA). A cluster was generated, diluted to $1.5 \mathrm{ng} / \mu \mathrm{L}$, and sequenced using the Illumina PE150 platform (Illumina, San Diego, CA) with paired-end $2 \times 150$-bp reads. Sequences were mapped to the chicken reference genome database from the NCBI database (ftp.ncbi.nlm.nih.gov/genomes/genbank/vertebrate_other/Gallus_gallus/latest_assembly_versions/GCA_000002315.5_GRCg6a/) with TopHat2 [42]. All RNA-seq data were uploaded to the NCBI Sequence Read Archive (https://www.ncbi.nlm.nih.gov/sra/PRJNA660090; SRA accession number: PRJNA660090).

\section{Protein extraction, TMT labeling and LC MS/MS analysis}

Total proteins from the same sample of RNA-seq were extracted by the cold acetone method. The supernatant from each sample, containing precisely $0.1 \mathrm{mg}$ of protein, was digested with Trypsin Gold (Promega, Madison, USA) at a 1:50 enzyme-to-substrate ratio. After $16 \mathrm{~h}$ of digestion at $37^{\circ} \mathrm{C}$, peptides were desalted with a $\mathrm{C} 18$ cartridge to remove the high urea, and desalted peptides were dried by vacuum centrifugation.

Desalted peptides were labeled with TMT6/10-plex reagents (TMT6/10plex ${ }^{T M}$ Isobaric Label Reagent Set, Thermo Fisher) following the manufacturer's instructions. Differently labeled peptides were mixed equally and subsequently desalted by peptide desalting spin columns (Thermo Fisher, 89852). TMT-labeled peptide mix was fractionated using a C18 column (Waters BEH C18 $4.6 \times 250 \mathrm{~mm}, 5 \mu \mathrm{m})$ on a Rigol L3000 HPLC operating at $1 \mathrm{~mL} / \mathrm{min}$, and the column oven was set at $50{ }^{\circ} \mathrm{C}$. Mobile phases A $(2 \%$ acetonitrile, adjusted $\mathrm{pH}$ to 10.0 using ammonium hydroxide) and $\mathrm{B}$ ( $98 \%$ acetonitrile, adjusted $\mathrm{pH}$ to 10.0 using ammonium hydroxide) were employed to develop a gradient elution. The eluates were monitored at UV $214 \mathrm{~nm}$, collected into a tube each minute and merged into 10 fractions. All fractions were dried under vacuum and reconstituted in $0.1 \%$ ( $v / v$ ) formic acid (FA) for subsequent analyses. 
Shotgun proteomics analyses were performed using an EASY-nLCTM 1200 UHPLC system (Thermo Fisher) coupled with an Orbitrap Q Exactive HF-X mass spectrometer (Thermo Fisher) operated in the data-dependent acquisition (DDA) mode.

Proteins with fold change in a comparison $\geq 1.2$ or $\leq 0.83$ and unadjusted significance level $p<0.05$ were considered to be differentially expressed. Gene Ontology (GO) and InterPro (IPR) analyses were conducted using the interproscan-5 program against the nonredundant protein database (including Pfam, PRINTS, ProDom, SMART, ProSiteProfiles, and PANTHER) [43], and the KEGG database (Kyoto Encyclopedia of Genes and Genomes) was employed to analyze the protein family and pathway. The probable interacting partners were predicted using the STRING-db server (http://string-db.org/) based on the related species. STRING is a database of both known and predicted protein-protein interactions [44]. The enrichment pipeline [45] was utilized to perform the enrichment analysis of GO and KEGG.

\section{Correlation analysis between proteomic and transcriptomic results}

The differentially expressed genes (DEGs) and the differentially expressed proteins (DEPs) were identified separately, and Venn diagrams were plotted according to the counted results. Correlation analysis was performed by R (version 3.5.1), and the maps were drawn based on changes in the transcriptome and proteome analyses.

\section{Quantitative real-time PCR analysis of differentially expressed genes}

RNA samples from the three chickens used for the RNA-seq experiment were analyzed by qRT-PCR. The concentrations of RNA samples were measured using a NanoQuant Plate (TECAN, Infinite M200PRO). Total cDNA was synthesized using a PrimeScript RT Reagent Kit with gDNA Eraser (Perfect Real Time) (TaKaRa, Dalian, China). qRT-PCR was performed with a LightCycle® 480 II (Roche) in a final volume of $20 \mu \mathrm{L}$ using THUNDERBIRD SYBR qPCR Mix (TOYOBO, Osaka, Japan). Each 20- $\mu \mathrm{L}$ reaction volume included $10 \mu \mathrm{L}$ of THUNDERBIRD SYBR qPCR Mix, $0.3 \mu \mathrm{L}$ of each divergent primer, $1 \mu \mathrm{L}$ of cDNA and $8.4 \mu \mathrm{L}$ of double-distilled $\mathrm{H}_{2} \mathrm{O}\left(\mathrm{ddH}_{2} \mathrm{O}\right)$. The cycling conditions included an initial single cycle $\left(98^{\circ} \mathrm{C}\right.$ for $\left.10 \mathrm{~s}\right)$ followed by 40 cycles $\left(94^{\circ} \mathrm{C}\right.$ for $15 \mathrm{~s}$ and $60{ }^{\circ} \mathrm{C}$ for $30 \mathrm{~s}$ ). $\beta$-actin was used as an internal control for the expression of mRNA. The relative expression level was normalized and calculated using the $2^{-\Delta \Delta C t}$ method [46]. All primers were designed using Primer 5 (listed in Supplementary Table S1).

\section{Western blot analysis}

RIPA lysis buffer (Beyotime, Beijing, China) was employed to generate uterine protein lysates. The uterine protein was extracted and separated using SDS-PAGE and transferred to PVDF membranes, which were blocked with skim milk. Then, antibodies specific for differential proteins (1:2000-1:1000, Abcam, Cambridge, MA, USA) and $\beta$-actin (1:1000, Abcam, Cambridge, MA, USA) were used for immunoblotting. We quantified the protein expression levels compared to $\beta$-actin expression using ImageJ $1.42 q$ (Wayne Rasband, National Institutes of Health, USA).

\section{Statistical analysis}

All data analyses were performed by GraphPad Prism 5 (San Diego, CA). The experimental values are presented as the means \pm SDs. The means of the groups were compared by Student's t-test. P-values $<0.05$ were considered to indicate significance.

\section{Abbreviations}

HE

hard-eggshell;

LE

week-eggshell;

DEGs

differentially expressed genes;

DEPs

differentially expressed proteins;

ANXA2

Annexin A2;

DKK3 
Dickkopf 3;

KRT14

Keratin-14

\section{Declarations}

\section{Consent for publication}

All authors have read and approved this version of the article. This manuscript has not been published in whole or in part nor is it being considered for publication elsewhere.

\section{Author Contributions}

YW and JP conceived of and designed the study; HZ, AP and HX performed the experiments; YP, JS and ZL collected the tissue samples and tested the egg quality; YW analyzed the data and wrote the manuscript; and JP and JD revised the manuscript.

\section{Availability of data and material}

The data that support the findings of this study are openly available in NCBI Sequence Read Archive at https://www.ncbi.nIm.nih.gov/sra/PRJNA660090, SRA reference number PRJNA660090. The mass spectrometry proteomics data have been deposited to the ProteomeXchange Consortium (http://proteomecentral.proteomexchange.org) via the iProX partner repository with the dataset identifier PXD021449.

\section{Competing interests}

The authors declare that they have no conflicts of interest.

\section{Funding}

This research was supported by the National Natural Science Foundation of China (32072709), Hubei Provincial Scientific and Technological Innovation Special Project (2019ABA084), China Agriculture Research System (CARS-40-S15), Leading Talents Program of Hubei Academy of Agricultural Sciences (L2018017).

\section{Acknowledgments}

We thank the anonymous reviewers for their critical reading and discussion of the manuscript.

\section{References}

1. Hincke MT, Nys Y, Gautron J, Mann K, Rodriguez-Navarro AB, McKee MD. The eggshell: structure, composition and mineralization. Front. Biosci. 2012; 17(1):1266..

2. Hrnčár, Cyril, Gič, Dominik, Bujko J . The Effect of Eggshell Quality on Hatchability of Pure Chicken Breeds. Scientific Papers: Animal Science \& Biotechnologies. 2012; 45(2): 415-18.

3. Park JA, Sohn SH. The influence of hen aging on eggshell ultrastructure and shell mineral components. Korean J Food Sci Anim Resour. 2018; 38: 1080-1091.

4. Vlčková J, Tůmová E, Míková K, Englmaierová M, Okrouhlá M, Chodová D. Changes in the quality of eggs during storage depending on the housing system and the age of hens. Poult Sci. 2019; 98: 6187-6193.

5. Roland DA. Egg shell problems: estimates of incidence and economicimpact. Poult Sci. 1988; 67: 1801-3.

6. Nys Y, Gautron J, Garcia-Ruiz JM, Hincke MT. Avian eggshell mineralization: Biochemical and functional characterization of matrix proteins. C. R. Palevol. 2004; 3:549-62.

7. Marie P, Labas V, Brionne A, Harichaux G, Hennequet-Antier C, Nys Y, et al. Data set for the proteomic inventory and quantitative analysis of chicken uterine fluid during eggshell biomineralization. Data Brief. 2014; 1:65-9. 
8. Marie P, Labas V, Brionne A, Harichaux G, Hennequet-Antier C, Nys Y, et al. Quantitative proteomics and bioinformatic analysis provide new insight into protein function during avian eggshell biomineralization. J Proteomics. 2015; 113: 178-93.

9. Carnarius KM, Conrad KM, Mast MG, Macneil JH. Relationship of eggshell ultrastructure and shell strength to the soundness of shell eggs. Poul Sci. 1996; 75: 656-63.

10. Ahmed AM, Rodriguez-Navarro AB, Vidal ML, Gautron J, García-Ruiz JM, Nys Y, Changes in eggshell mechanical properties, crystallographic texture and in matrix proteins induced by moult in hens. Br Poult Sci. 2005; 46:268-79.

11. Zhang YN, Zhang HJ, Wu SG, Wang J, Qi GH. Dietary manganese supplementation modulated mechanical and ultrastructural changes during eggshell formation in laying hens. Poult Sci. 2017; 96: 2699-707.

12. Stefanello C, Santos TC, Murakami AE, Martins EN, Carneiro TC. Productive performance, eggshell quality, and eggshell ultrastructure of laying hens fed diets supplemented with organic trace minerals. Poult Sci. 2014; 93: 104-13.

13. Nys Y, Hincke M, Hernandez-Hernandez A, Rodriguez-Navarro A, Gomez-Morales J, Jonchere V, et al. Eggshell ultrastructure, properties and the process of mineralization: involvement of organic matrix in the eggshell fabric. Productions AnimalesParis- Institut National de la Recherche Agronomique-. 2010; 23:143-154.

14. Nys Y, Gautron J, Garcia-Ruiz JM, Hincke MT. Avian eggshell mineralization: biochemical and functional characterization of matrix proteins, Comptes rendus - Palevol. 2004; 3: 549-62.

15. Hincke MT, Gautron JL, Charles PWT, McKee MD, Nys Y. Molecular cloning and ultrastructural localization of the core protein of an eggshell matrix proteoglycan, Ovocleidin-116. J Biol Chem. 1999; 274(46):32915-23.

16. Hincke MT, Tsang C, Courtney M, Hill V, Narbaitz R. Purification and immunochemistry of a soluble matrix protein of the chicken eggshell (ovocleidin 17). Calcif Tissue Int. 1995; 56(6): 578-83.

17. Gautron J, Hincke MT. Ovocalyxin-32, a novel chicken eggshell matrix protein - isolation, amino acid sequencing, cloning, andimmunocytochemical localization. J Biol Chem. 2001; 276(42): 39243-52.

18. Gautron J, Murayama E, Vignal A, Morisson M, McKee MD, Réhault S, et al Cloning of Ovocalyxin-36, a novel chicken eggshell protein related to lipopolysaccharide-binding proteins, bactericidal permeability-increasing proteins, and plunc family proteins. J Biol Chem. 2007; 282(8): 5273-86.

19. Mann K. The calcified eggshell matrix proteome of a songbird, the zebra finch (Taeniopygia guttata). Proteome Sci. 2015; 13 : 29.

20. Crosara F, Pereira VJ, Lellis CG, Barra KC, Fernandes EA. Is the Eggshell Quality Influenced by the Egg Weight or the Breeder Age? Revista Brasileira de Ciência Avícola. 2019; 10.1590/1806-9061-2018-0896.

21. Sirri F, Zampiga M, Berardinelli A, Meluzzi A. Variability and interaction of some egg physical and eggshell quality attributes during the entire laying hen cycle, Poult Sci., 2018; 97: 1818-23.

22. Bain MM, Nys Y, Dunn IC. Increasing persistency in lay and stabilising egg quality in longer laying cycles. What are the challenges? Br Poult Sci. 2016; 57: 330-8.

23. Li LL, Zhang NN, Gong YJ, Zhou MY, Zhan HQ, Zou XT. Effects of dietary Mn-methionine supplementation on the egg quality of laying hens. Poult Sci. 2017; doi: 10.3382/ps/pex301.

24. Carnarius KM, Conrad KM, Mast MG, Macneil JH. Relationship of eggshell ultrastructure and shell strength to the soundness of shell eggs. Poult Sci. 1996; 75: 656-63.

25. S. E. Solomon, Egg and Eggshell Quality, Wolfe, London, UK. 1991.

26. Hunton P. Research on eggshell structure and quality: An historical overview. Br J Poult Sci. 2005; 7: 67-71.

27. Bain MM. Eggshell strength: A relationship between the mechanism of failure and the ultrastructural organisation of the mammillary layer. Br Poult Sci. 1992; doi: 10.1080/00071669208417469.

28. Fathi MM, Zein El-Dein A囚EI-Safty SA囚Lamiaa M. Radwan. Using scanning electron microscopy to detect the ultrastructural variations in eggshell quality of Fayoumi and Dandarawi chicken breeds. Int J Poult Sci. 2007; doi:

10.3923/ijps.2007.236.241.

29. Rodriguez-Navarro A, Kalin O, Nys Y, Garcia-Ruiz JM. Influence of the microstructure on the shell strength of eggs laid by hens of different ages. Brit Poult Sci. 2002; 433(3): 395-403.

Page 9/16 
30. Meyer R, Baker RC, Scott ML. Effects of hen egg-shell and other calcium sources upon egg-shell strength and ultrastructure. Poult Sci. 1973; 52(3): 949-55.

31. Brionne A, Nys Y, Hennequet-Antier C, Gautron J. Hen uterine gene expression profiling during eggshell formation reveals putative proteins involved in the supply of minerals or in the shell mineralization process. BMC Genomics. 2014; 15: 220.

32. Jonchère V, Réhault-Godbert S, Hennequet-Antier C, Cabau C, Sibut V, Cogburn LA, et al. Gene expression profiling to identify eggshell proteins involved in physical defense of the chicken egg. BMC Genomics. 2020; 9: 1081-4.

33. Johnson MD, Kenney N, Stoica A, Hilakivi-Clarke L, Singh B, Chepko G, et al. Cadmium mimics the in vivo effects of estrogen in the uterus and mammary gland. Nat Med. 2003; 120: 284-289.

34. Kippler M, Tofail F, Gardner R, Rahman A, Hamadani JD, Bottai M, et al. Maternal cadmium exposure during pregnancy and size at birth: a prospective cohort study. Environ Health Perspect. 2012; 20(1):707.

35. Zhang J, Wang Y, Zhang C, Xiong M, Rajput SA, Liu Y, et al. The differences of gonadal hormones and uterine transcriptome during shell calcification of hens laying hard or weak-shelled eggs. BMC Genomics. 2019; 20(1):707.

36. Ma Q, Shi C, Su C, Liu Y. Complementary analyses of the transcriptome and iTRAQ proteome revealed mechanism of ethylene dependent salt response in bread wheat (Triticum aestivum L.). Food Chemistry. 2020; 325:126866.

37. Ramirez-Carrozzi VR, Nazarian AA, Li CC, Gore SL, Sridharan R, Imbalzano AN, et al. Selective and antagonistic functions of SWI/SNF and Mi-2beta nucleosome remodeling complexes during an inflammatory response. Genes Dev. 2006; 20(3):28296.

38. Smale ST. Selective transcription in response to an inflammatory stimulus. Cell. 2020; 140(6):833-44..

39. Dallacasagrande V, Hajjar KA. Annexin A2 in Inflammation and Host Defense. Cells. 2020; 9(6): 1499.

40. Fujita H, Bando T, Oyadomari S, Ochiai K, Watanabe M, Kumon H, et al. Dkk3/REIC, an N-glycosylated Protein, Is a Physiological Endoplasmic Reticulum Stress Inducer in the Mouse Adrenal Gland. Acta Med Okayama. 2020; 74(3):199-208.

41. Bilandzic M, Rainczuk A, Green E, Fairweather N, Jobling TW, Plebanski M, et al. Keratin-14 (KRT14) Positive Leader Cells Mediate Mesothelial Clearance and Invasion by Ovarian Cancer Cells. Cancers (Basel). 2019; 11(9): 1228.

42. Dou Y, Cha DJ, Franklin JL, Higginbotham JN, Jeppesen DK, Weaver AM, et al. Circular RNAs are down-regulated in KRAS mutant colon cancer cells and can be transferred to exosomes. Sci Rep. 2016; 6: 37982.

43. Jones P, Binns D, Chang HY, Fraser M, Li W, McAnulla C, et al. InterProScan 5: genome-scale protein function classification. Bioinformatics. 2014; 30: 1236-40.

44. Franceschini A, Szklarczyk D, Frankild S, Kuhn M, Simonovic M, Roth A, et al. STRING v9.1: protein-protein interaction networks, with increased coverage and integration. Nucleic Acids Res. 2013; doi: 10.1093/nar/gks1094.

45. Huang da W, Sherman BT, Lempicki RA. Bioinformatics enrichment tools: paths toward the comprehensive functional analysis of large gene lists. Nucleic Acids Res. 2009; doi: 10.1093/nar/gkn923.

46. Livak KJ, Schmittgen TD. Analysis of relative gene expression data using real-time quantitative PCR and the $2^{-\triangle \Delta C T}$ method. Methods. 2001; 25: 402-8.

\section{Figures}




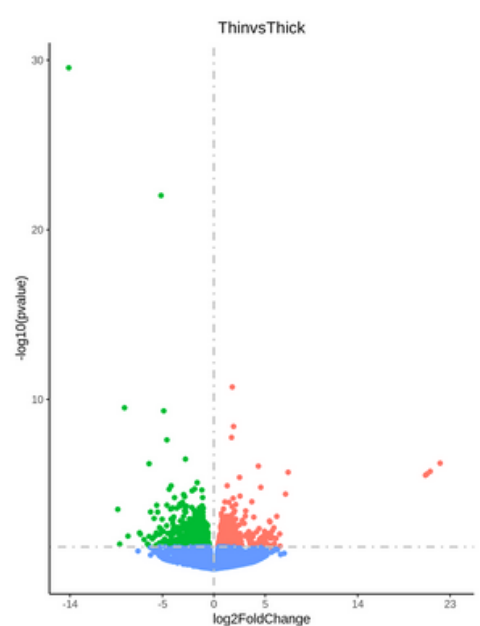

A

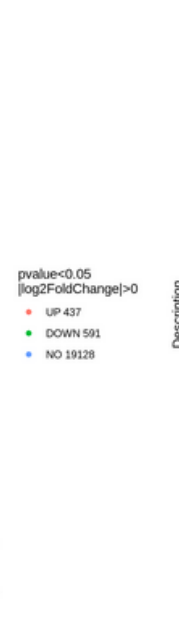

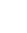

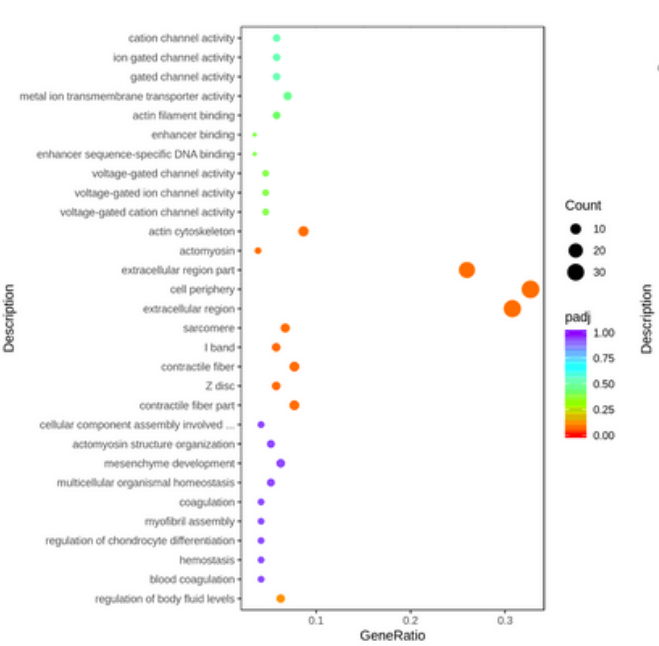

B

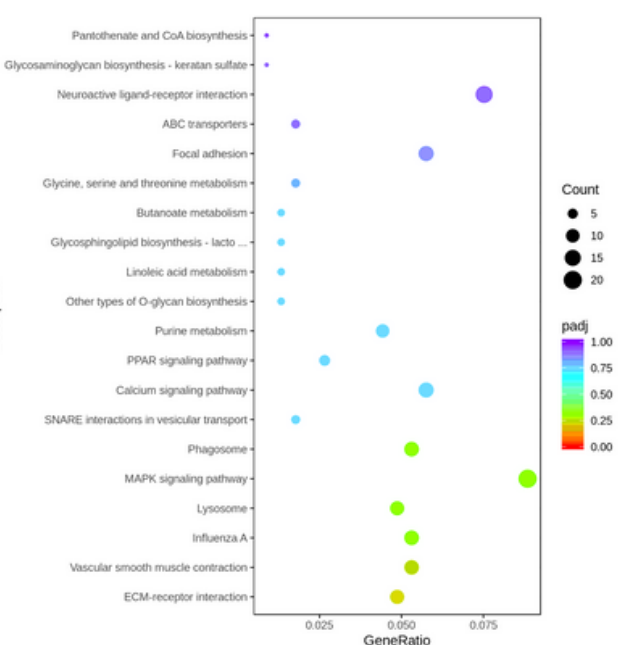

C

\section{Figure 1}

Differentially expressed genes (DEGs) identified for uterus of HE and LE hens. a. Volcano plot of DEGs in the uterus; $b$. GO analysis of DEGs between HE and LE hens; C. KEGG analysis of DEGs between HE and LE hens.

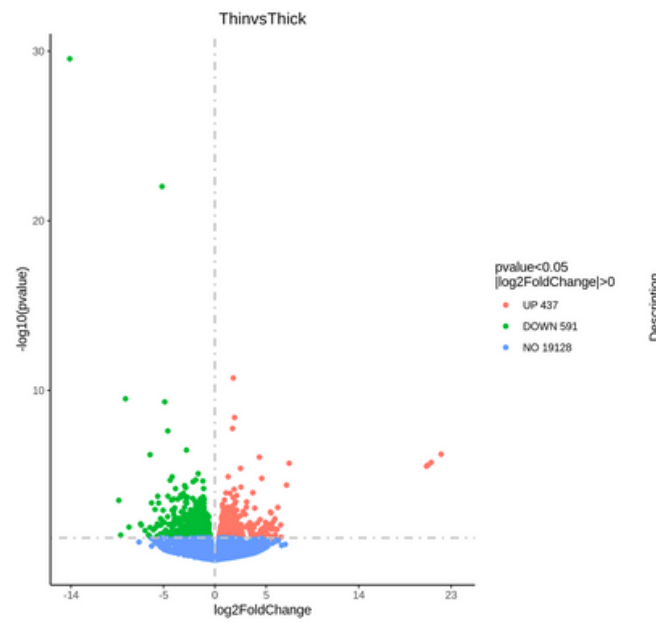

A

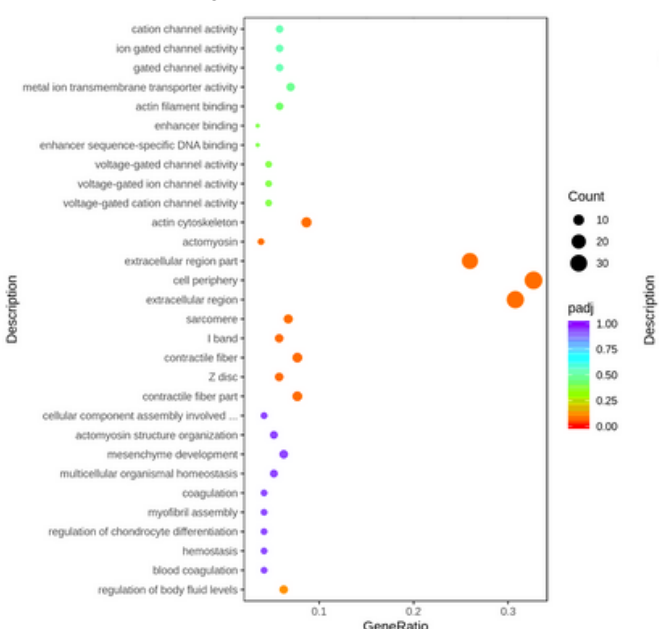

B

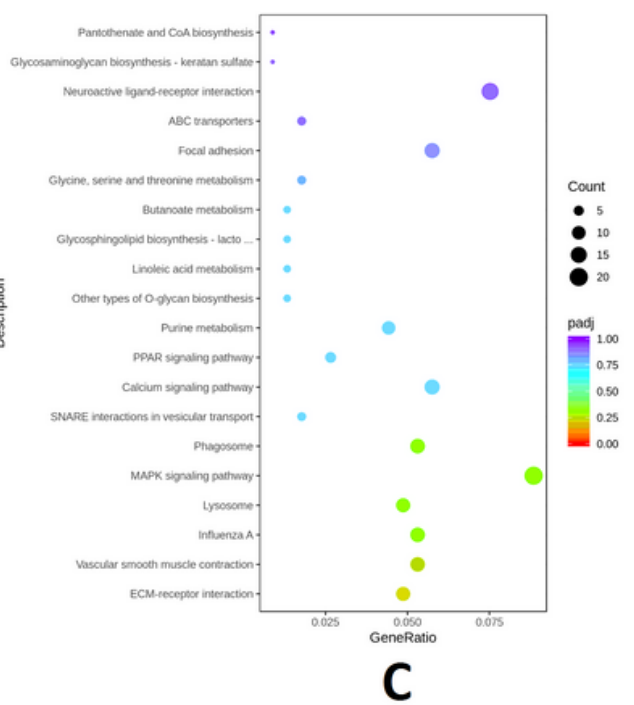

Figure 1

Differentially expressed genes (DEGs) identified for uterus of HE and LE hens. a. Volcano plot of DEGs in the uterus; b. GO analysis of DEGs between HE and LE hens; C. KEGG analysis of DEGs between HE and LE hens. 

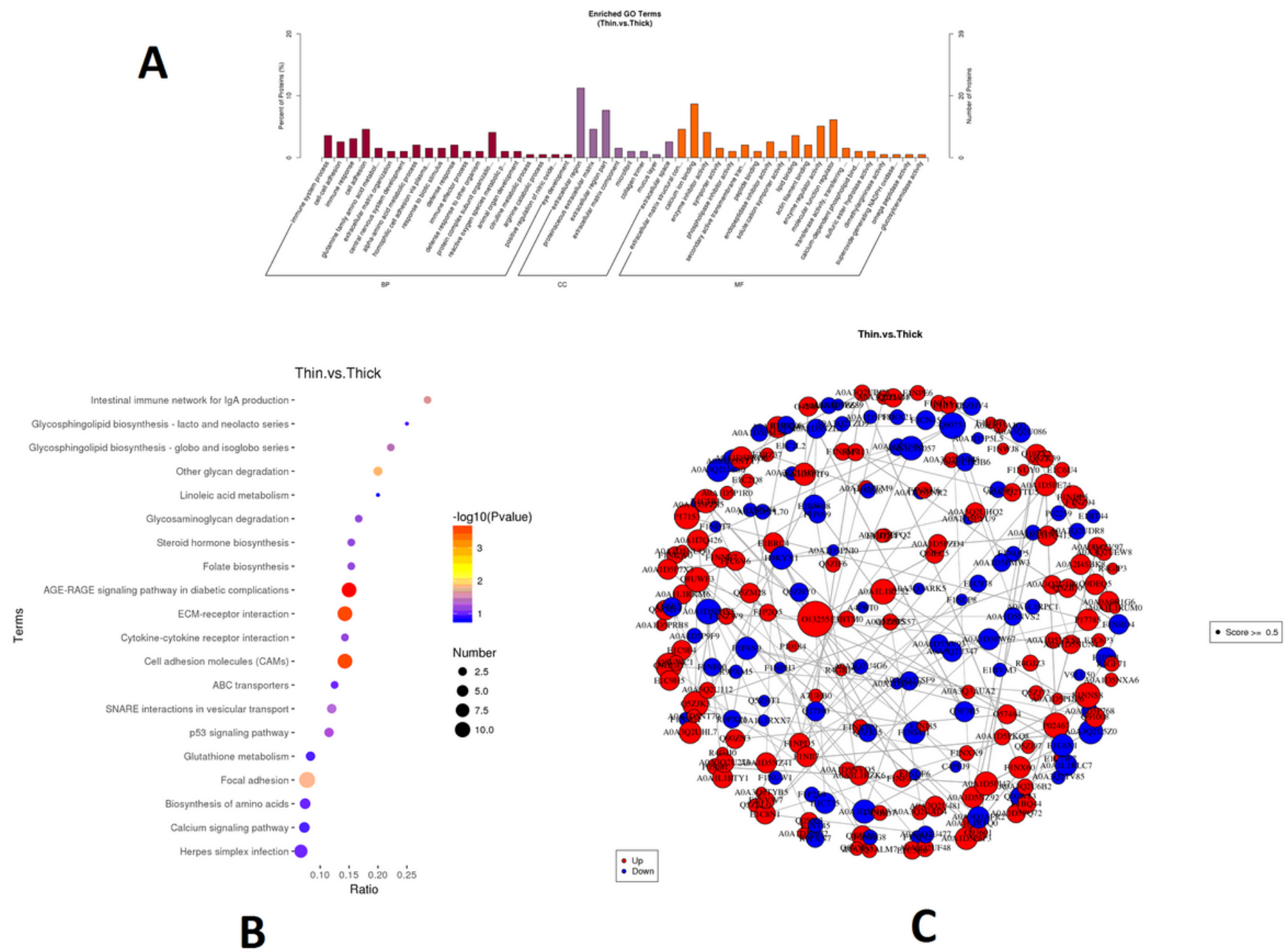

Figure 2

Differentially expressed proteins (DEGs) identified for uterus of HE and LE hens. a. GO analysis of DEPs between HE and LE hens; b. The top 20 results of KEGG analysis of DEPs between HE and LE hens; c. The protein-protein interaction network of the DEPs. 

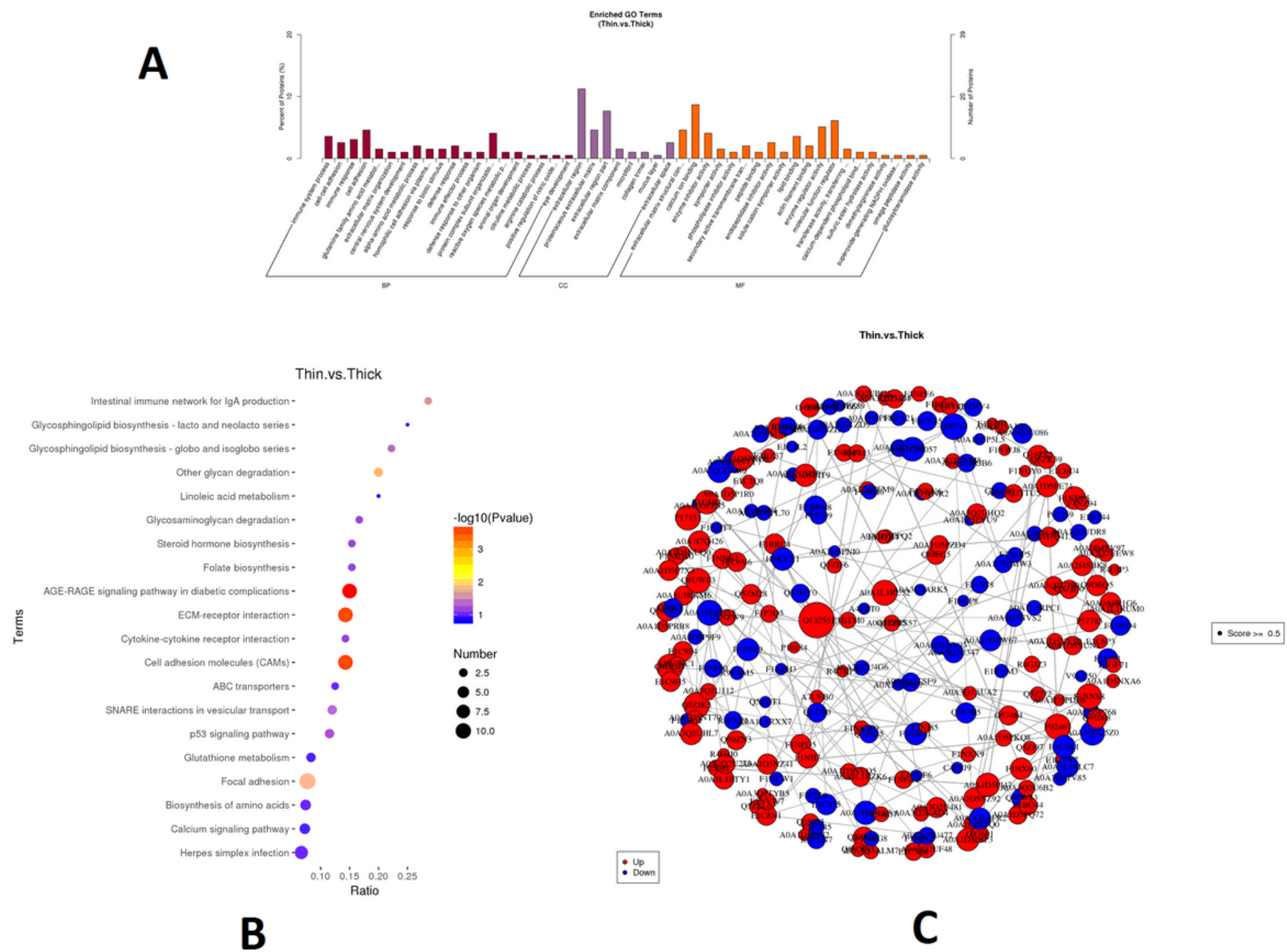

Figure 2

Differentially expressed proteins (DEGs) identified for uterus of HE and LE hens. a. GO analysis of DEPs between HE and LE hens; b. The top 20 results of KEGG analysis of DEPs between HE and LE hens; c. The protein-protein interaction network of the DEPs. 

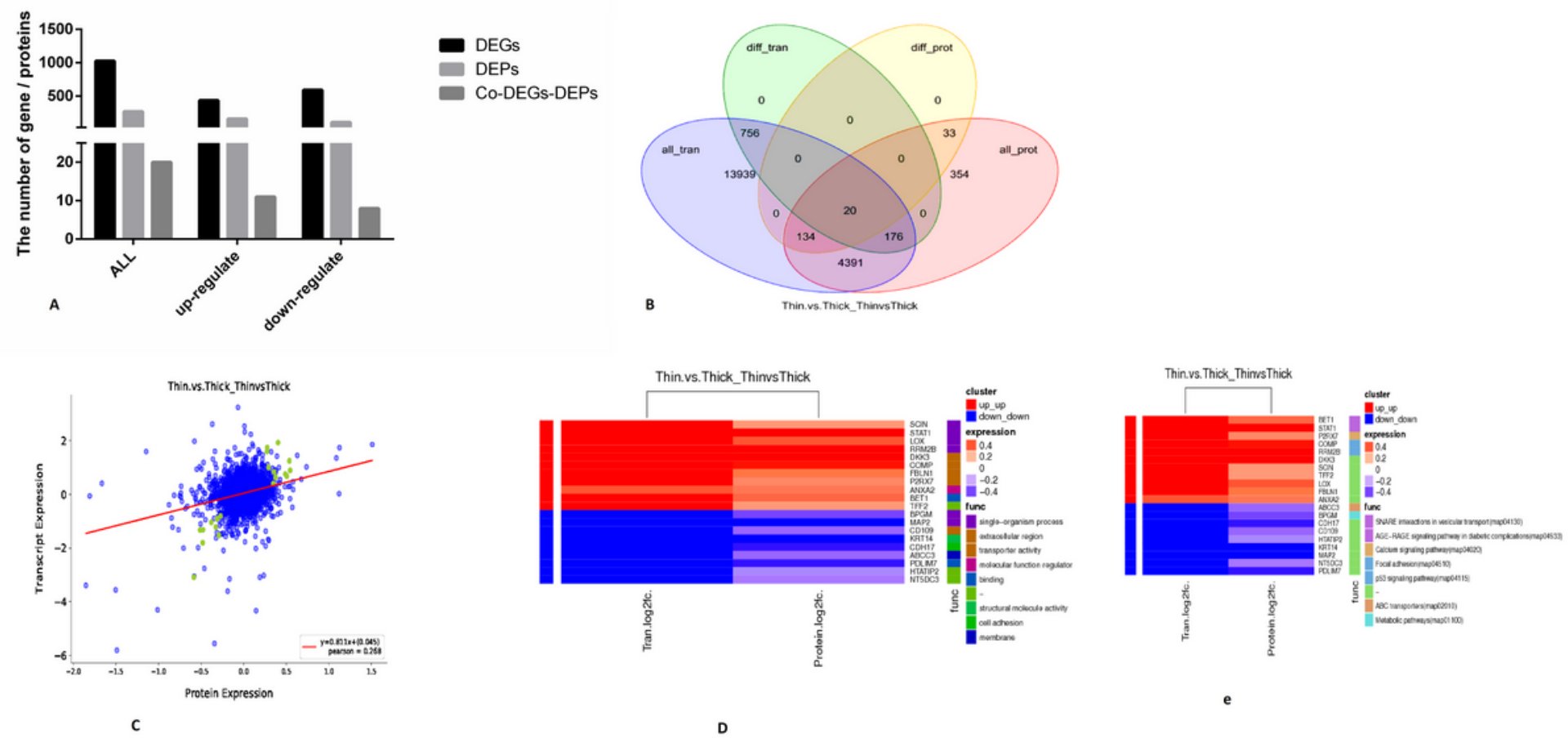

Figure 3

The integrated and analyzed of transcriptome and proteome in the HE and LE groups. a. The DEGs, DEPs or Co-DEGs-DEPs between HE and LE groups; b. An over Venn diagram showed the overlap among genes, proteins, DEGs and DEPs; $c$. The corr_plot of transcriptome and proteome between HE and LE groups; $d$. The share $\mathrm{GO}$ analysis of transcriptome and proteome between HE and LE groups; e. The share KEGG analysis of transcriptome and proteome between HE and LE groups.
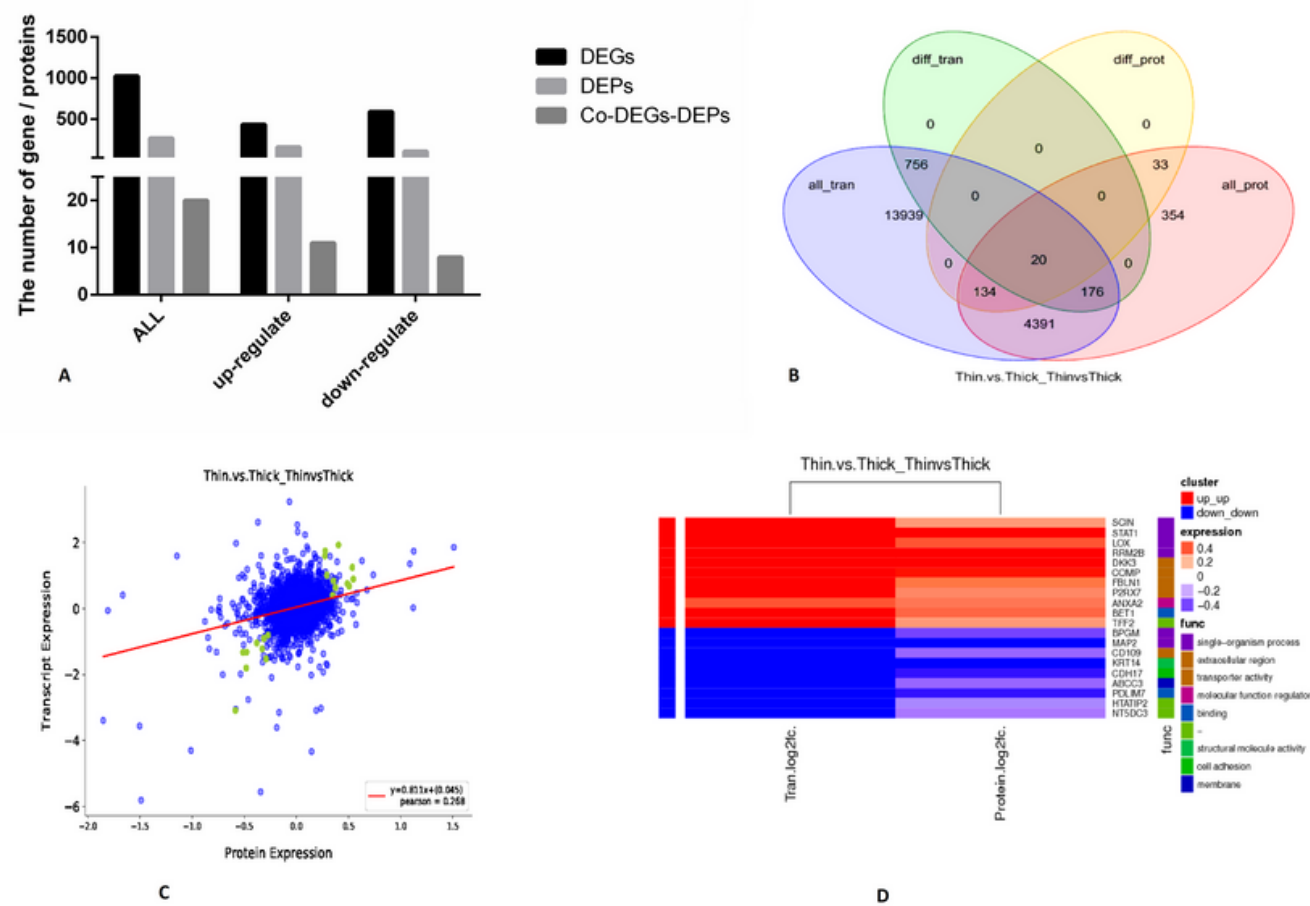

Thin. .Thick_ThinusThick
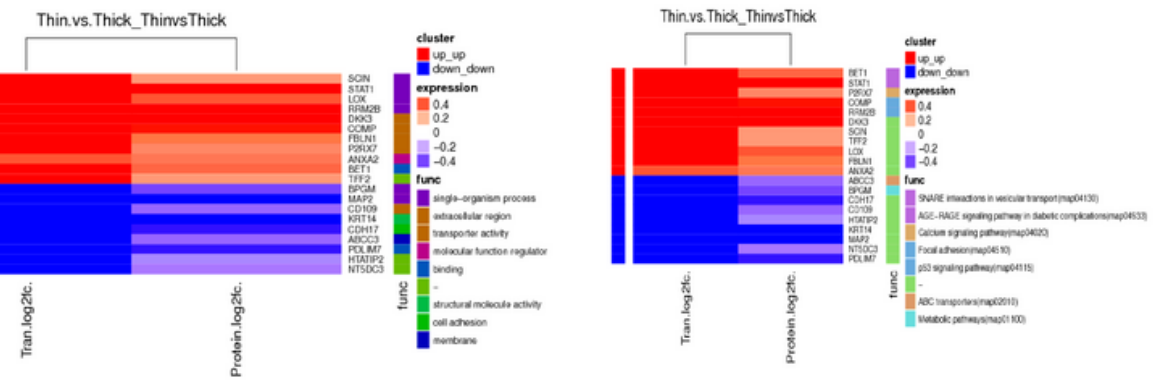

Figure 3

The integrated and analyzed of transcriptome and proteome in the HE and LE groups. a. The DEGs, DEPs or Co-DEGs-DEPs between HE and LE groups; b. An over Venn diagram showed the overlap among genes, proteins, DEGs and DEPs; c. The corr_plot of transcriptome and proteome between HE and LE groups; $d$. The share GO analysis of transcriptome and proteome between $\mathrm{HE}$ and LE groups; e. The share KEGG analysis of transcriptome and proteome between HE and LE groups. 

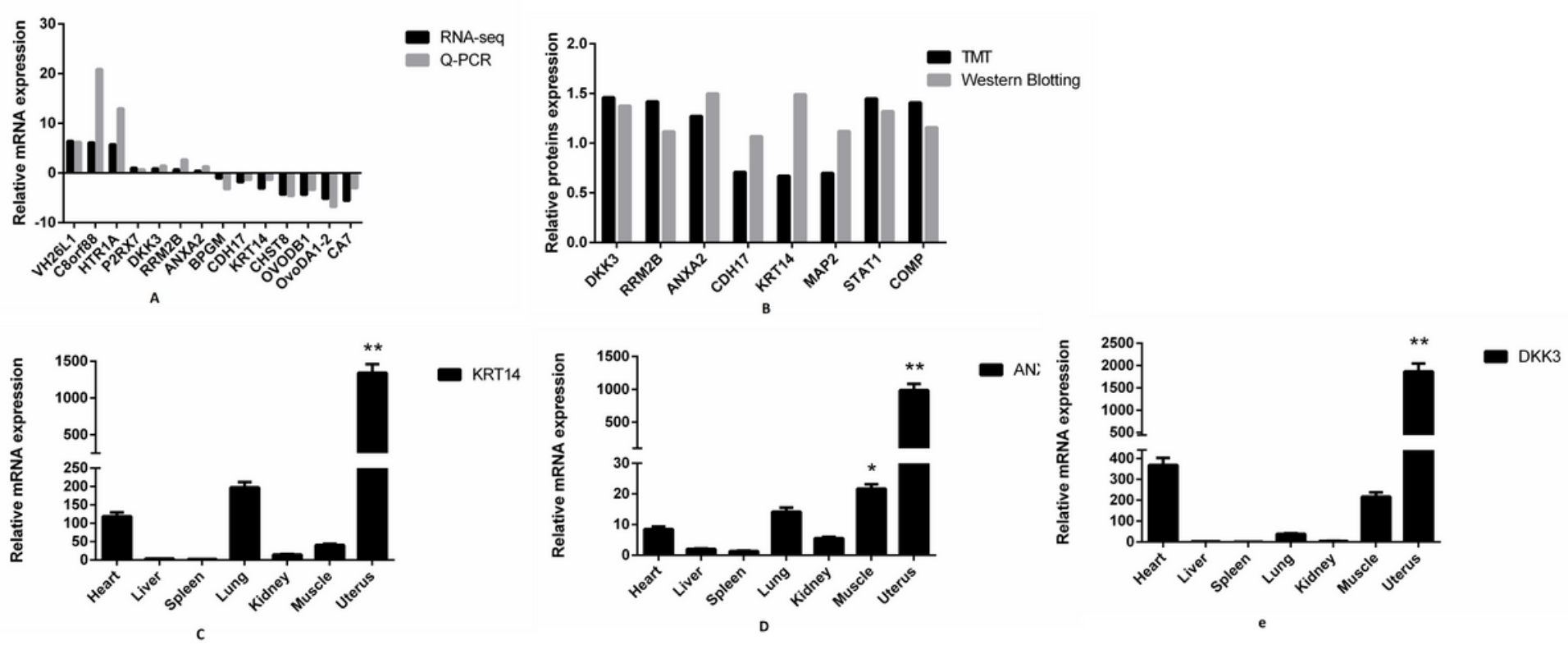

Figure 4

Differentially expressed gene and protein identification and verification. a. Changes in DEGs expression validated by qRT-PCR. Comparison results of the 14 mRNAs using the qRT-PCR and RNA-seq; $b$. Changes in DEPs expression validated by Western Blotting. Comparison results of the 8 proteins using the Western Blotting and TMT; c. Relative expression of KRT14 in different tissures; d. Relative expression of ANXA2 in different tissures; e. Relative expression of DKK3 in different tissures.
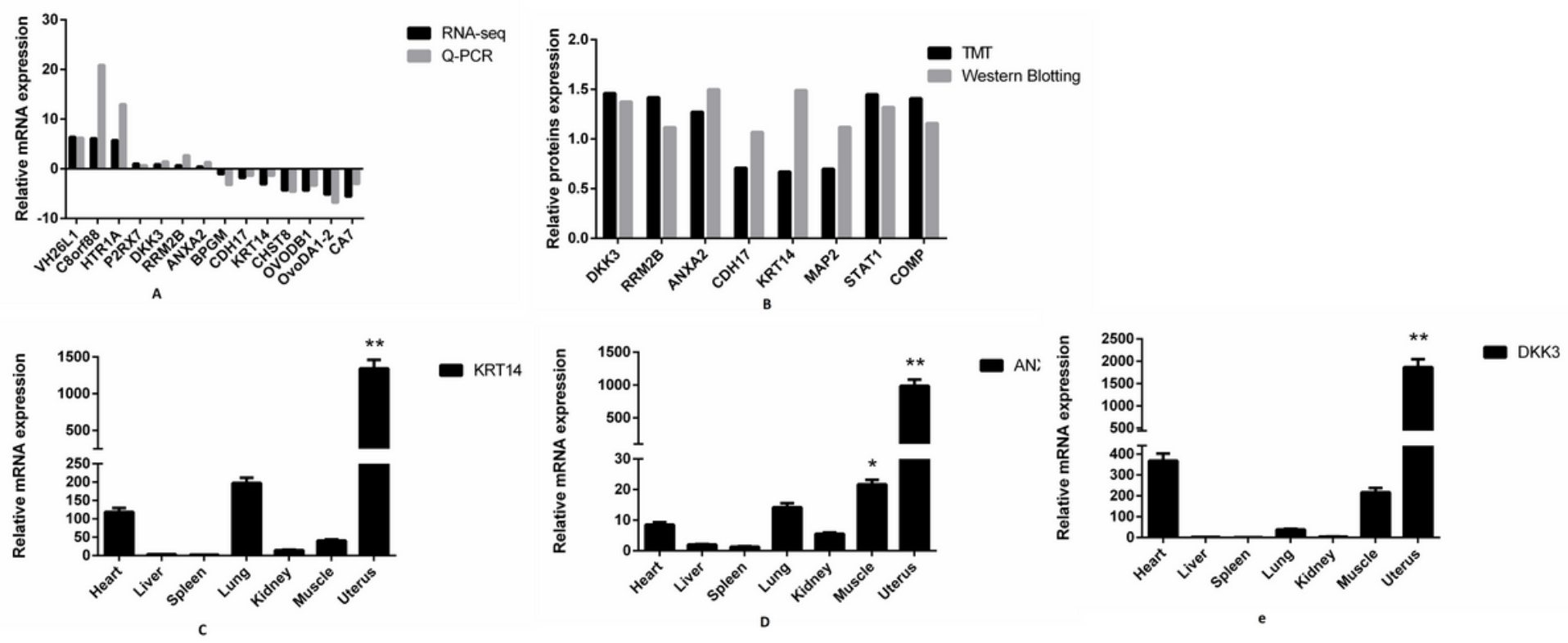

Figure 4

Differentially expressed gene and protein identification and verification. a. Changes in DEGs expression validated by qRT-PCR. Comparison results of the 14 mRNAs using the qRT-PCR and RNA-seq; b. Changes in DEPs expression validated by Western Blotting. Comparison results of the 8 proteins using the Western Blotting and TMT; c. Relative expression of KRT14 in different tissures; d. Relative expression of ANXA2 in different tissures; e. Relative expression of DKK3 in different tissures.

\section{Supplementary Files}

This is a list of supplementary files associated with this preprint. Click to download. 
- SupplementaryTableS1.docx

- SupplementaryTableS1.docx

- SupplementalTableS2.docx

- SupplementalTableS2.docx

- SupplementalTableS3.xlsx

- SupplementalTableS3.xIsx

- SupplementalTableS4.xIsx

- SupplementalTableS4.xlsx 